\title{
28 Research Square \\ Validity of Mechanical advantage hypothesis of human grasping depends on the nature of task difficulty
}

\section{Banuvathy Rajakumar}

Indian Institute of Technology Madras

\section{Swarnab Dutta}

Indian Institute of Technology Madras

Varadhan SKM ( $\nabla$ skm@iitm.ac.in )

Indian Institute of Technology Madras

\section{Research Article}

Keywords: Grasping, Perturbation, Mechanical advantage

Posted Date: November 8th, 2021

DOI: https://doi.org/10.21203/rs.3.rs-1058248/v1

License: (c) (1) This work is licensed under a Creative Commons Attribution 4.0 International License.

Read Full License 


\section{Abstract}

Successful object interaction during daily living involves maintaining the grasped object in a static equilibrium by properly arranging the fingertip contact forces. According to the mechanical advantage hypothesis, during supination or pronation torque production, fingers with longer moment arms would produce greater normal force than those with shorter moment arms. Previous studies have probed this hypothesis by investigating the force contributions of the individual fingers through systematic variations (or perturbations) of properties of the grasped handle. In the current study, we examined the applicability of this hypothesis in a paradigm wherein the thumb tangential force was constrained to a minimal constant magnitude. This was achieved by placing the thumb on a freely movable slider platform. The total mass of the handle was systematically varied by adding external loads directly below the center of mass of the handle. Our findings suggest that in the human hand, the central nervous system (CNS) adopts the principle of mechanical advantage depending on an abstract sense of challenge attached to the task situation.

\section{Introduction}

Among all the activities of daily living, grasping and manipulating objects is one of the most crucial and fundamental actions performed by humans. Maintaining the grasped object in static equilibrium is perhaps a critical aspect that governs safe handling by avoiding clumsy behavior such as dropping and spilling. Any form of perturbations to the grasped object requires a complete rearrangement of the fingertip forces to sustain the static equilibrium.

Previously, studies have been performed to investigate the force contribution of the individual fingers and thumb when systematic variations (or perturbations) were imparted to the properties of the grasped handle. Such variations involved introducing external torques ${ }^{1-4}$, varying the mass of the external loads ${ }^{5,6}$, surface friction modification ${ }^{7-9}$, alteration to the grip width of the handle ${ }^{10}$ or individual digit width ${ }^{11}$ in the horizontal direction, and change in the position of the fingertips ${ }^{12}$ and the thumb ${ }^{3}$ while grasping. Normal and tangential forces of the individual fingers and thumb varied systematically in response to these perturbations. Some of these perturbations also disturbed the rotational equilibrium of the handle. In such situations, compensatory torque production was required by the fingers to sustain the handle in static equilibrium. During the compensatory supination or pronation torque production, according to the mechanical advantage hypothesis $(\mathrm{MAH})^{13,14}$, it was expected that the fingers with longer moment arms for normal force would produce greater normal force than those with the shorter moment arms.

Several studies have attempted to examine the applicability of the mechanical advantage principle for a five-finger grasping task. Mechanical advantage hypothesis was supported in tasks that involved handle rotation in the pronation and supination directions at two different speeds ${ }^{15}$, the addition of an external load of different masses at varying distances from the center of mass of the handle ${ }^{16}$, and moment production to follow a trapezoid template by pressing with all four fingers ${ }^{17}$. However, the hypothesis was 
only partially supported in a moment production task on a mechanically fixed object ${ }^{18}$, where the distance from the fingers to the axis of rotation, magnitude, and direction of torque production was varied systematically. The authors of the afore-mentioned study posited that the applicability of the MAH may be task and effector-specific. As such, it is yet unclear what kind of tasks the applicability of MAH depends on. Therefore, it is necessary to investigate whether the applicability of mechanical advantage is task-specific and which kind of tasks/scenarios support the MAH.

In our previous study ${ }^{19}$, we had attempted to investigate the applicability of MAH by introducing torque changes to the handle. Rather than implementing external torque changes by suspending the load at a distance from the center of mass of the handle, we incorporated the torque changes by reducing friction between the thumb platform and the handle interface. This was made possible by placing the thumb on a slider platform that could freely translate vertically over a railing. In this way, the tangential force produced by the thumb was kept constant and less than the virtual finger ${ }^{20}$ (an imaginary finger whose mechanical output is equal to the combined output of the individual fingers except for the thumb). This had resulted in introducing a residual pronation torque to the handle. Since the instruction was to maintain the handle in static equilibrium, a compensatory supination torque was required to avoid the tilt caused due to the residual pronation torque. Ulnar finger normal forces and thumb tangential forces are major contributors to this compensatory supination torque. However, by our design, it was not possible to increase the tangential force of the thumb as it had to hold the slider platform steady at the HOME position (midway between middle and ring fingers). Therefore, only the normal forces produced by the ulnar fingers became the primary source of this compensatory supination torque.

Between the ulnar fingers, the little finger has a larger moment arm for normal force when compared with the ring finger. Hence it was expected that the little finger would produce greater normal force. Contrary to this expectation, ring and little fingers were found to share comparable normal forces while grasping the handle of mass $0.535 \mathrm{~kg}$. Therefore, in the current study, we expected that MAH would be corroborated if the mass of the handle is increased systematically by adding different external loads. As per the design of this grip device, the tangential force of the thumb was constrained to a constant minimal magnitude. So, with an increase in the mass of the handle, only the tangential force of the virtual finger increases, which is accompanied by an increase in the residual pronation torque. As a corrective effect, the magnitude of compensatory supination torque required to be produced would also increase. Hence, we expected that with a systematic increase in the mass of the handle, the little finger would produce correspondingly higher normal force than the ring finger during compensatory supination torque production.

In line with such an expectation, in the current study, the mass of the handle was systematically increased by employing external loads of mass $0.150,0.250,0.350$, and $0.450 \mathrm{~kg}$ as different experimental conditions. Our previous study ${ }^{19}$ had shown comparable normal forces between the ulnar fingers for a handle mass of $0.535 \mathrm{~kg}$. In another study on investigating the role of grasp force magnitude during multi-finger prehension ${ }^{21}$, by suspending an external load of mass $0.160 \mathrm{~kg}$ eccentrically at various 
distances under a handle of mass $0.415 \mathrm{~kg}$, the contribution of digit forces in terms of percentage of total normal force of the virtual finger was examined. It was found that even for a small external torque of 0.14 $\mathrm{Nm}$, during natural grasping, the percentage share of the little finger normal force (approx. 45\%) was greater than the ring finger normal force (approx. 33\%).

The total mass of the handle $(0.450 \mathrm{~kg})$ with the minimum external load $(0.150 \mathrm{~kg})$ used in our current study was approximately close to the total mass of the grip device in the afore-mentioned multi-finger prehension study ${ }^{21}$. Hence, we hypothesized that the mechanical advantage hypothesis would be supported for all experimental conditions $(0.150 \mathrm{~kg}, 0.250 \mathrm{~kg}, 0.350 \mathrm{~kg}$, and $0.450 \mathrm{~kg})$ of external load starting with a minimal mass of $0.150 \mathrm{~kg}$ (Hypothesis $\mathrm{H} 1$ ).

\section{Methods And Materials}

\section{Participants}

Twelve young, healthy right-handed male volunteers participated in this study. The mean and standard deviation of the participant's age, height, weight, hand length, and width were measured as follows: Age: $26.75 \pm 3.9$ years, Height: $172.02 \pm 5.7 \mathrm{~cm}$, Weight: $75.21 \pm 17.7 \mathrm{~kg}$, Hand-length: $18.93 \pm 1.1 \mathrm{~cm}$, and Handwidth: $8.92 \pm 0.7 \mathrm{~cm})$. Only participants with no history of neurological diseases and musculoskeletal injuries were chosen to participate in this experiment.

\section{Ethics Approval}

The Institutional Ethics committee of the Indian Institute of Technology Madras approved the experimental procedures (Approval Number: IEC/2021-01/SKM/02/05). All the participants gave written informed consent according to the procedure approved by the institutional ethics committee of IIT Madras before the beginning of the experiment. The experimental sessions were conducted by strictly adhering to the procedures approved by the Institutional Ethics Committee of the Indian Institute of Technology Madras.

\section{Experimental setup}

A five-finger prehensile handle was designed and custom-built for the experiment, as shown in Fig.1. The handle consists of a vertical railing of length $13.6 \mathrm{~cm}$ fitted on the thumb side to mount the slider platform, thus allowing its vertical translation along the railing. The handle was suspended from wooden support using a nylon rope housed within a hollow PVC pipe to restrict any undesirable lateral movement while it was suspended. The present study involves a prismatic precision grip of the handle of mass $0.450 \mathrm{~kg}$. The mass of the slider platform was $0.100 \mathrm{~kg}$. Thus, restricting the thumb tangential force to approximately $1 \mathrm{~N}$. Five six-axis force/torque sensors (Nano 17, Force resolution: Tangential: $0.0125 \mathrm{~N}$, Normal: $0.0125 \mathrm{~N}$, ATI Industrial Automation, NC, USA) was mounted on the handle to measure the forces 
and the moments exerted by the individual fingers and thumb. For the thumb alone, the force sensor was placed on the slider platform, which enabled the smooth translation of the platform over the railing fitted on the handle's thumb side.

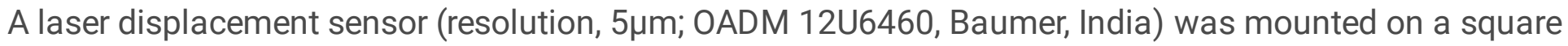
flat piece made of acrylic, and the assembly was fitted on top of the handle towards the thumb side. The displacement sensor provided the displacement data of the thumb platform in the vertical direction while it translated along the vertical railing. On top of the handle, another acrylic block was placed in the anterior-posterior direction, which held an intelligent 9-axis absolute orientation sensor (Resolution: 16bits, Range: 2000\% s, Model: BNO055, BOSCH, Germany). This IMU (Inertial Measurement Unit) sensor provided the orientation data of the handle after appropriate pre-processing of the raw data. A spirit level was also mounted on the acrylic block towards the participant's side of the handle to aid the participant in ensuring the handle's vertical orientation while it was being held.

Two horizontal lines were drawn on the participant's side of the handle, one at the center of the thumb platform and another midway between middle and ring fingers on the handle frame. The participants were asked to hold the handle in a way such that the two lines were aligned. Thirty analog signals from the force/torque sensors ( 5 sensors $\times 6$ components) and single-channel analog laser displacement data were digitized using NI USB 6225 and 6002 at 16-bit resolution (National Instruments, Austin, TX, USA). This data was synchronized with four channels of processed, digital data from the IMU sensor. Sampling rates of all data were set to $100 \mathrm{~Hz}$.

\section{Experimental procedure}

Participants were asked to wash and clean their hands with soap, towel-dry and then sit comfortably on a wooden chair with their forearm resting on the table. The right upper arm was abducted at approximately $45^{\circ}$ in the frontal plane, flexed $45^{\circ}$ in the sagittal plane, and elbow flexed at approximately $90^{\circ}$. The natural grasping position can be achieved by supinating the forearm at $90^{\circ}$. The movements of the forearm and wrist were constrained by fastening with a Velcro strap to the tabletop.

The experiment involved four conditions. For these conditions, external loads of mass $0.150 \mathrm{~kg}, 0.250 \mathrm{~kg}$, $0.350 \mathrm{~kg}$, and $0.450 \mathrm{~kg}$ were added at the bottom of the handle, i.e., exactly under the center of mass of the handle. A computer monitor displayed a solid horizontal target line with two dashed lines at $0.2 \mathrm{~cm}$ above and below the target line. These dashed lines represented an acceptable error margin. The target line shown on the monitor corresponded to the 'HOME position' of the thumb. The trial began only after the participant could hold the thumb platform steadily by aligning the horizontal line on the thumb platform to the line drawn midway between the middle and ring finger. Thumb displacement data measured using

a laser displacement sensor was shown as feedback on the participant's screen. Once the trial started, the participants were required to keep the slider platform in the same position (HOME), by aligning the horizontal line on the platform to the line drawn between the middle and the ring fingers. Precise 
alignment of the two lines during the task essentially meant that the feedback line traced the actual target line. Acceptable performance or task success during the trial was defined to be within an error margin of $\pm 0.2 \mathrm{~cm}$ as mentioned above. Throughout the trial, the handle had to be maintained in static equilibrium in the frontal plane for all the external loads. This was ensured by having the bubble of the spirit level at the center throughout the trial.

For each experimental condition, 25 trials were performed. Each trial lasted for six seconds. One minute of break was provided between trials. After every twelve trials, ten minutes of break was provided to eliminate the effect of fatigue, if any. The experiment was held in two separate sessions. Each session included two external load conditions with thirty minutes of break between conditions. The order of presentation of these two sessions was counterbalanced across all participants. Six of the participants performed with the weight of $0.150 \mathrm{~kg}$ followed by $0.350 \mathrm{~kg}$ in their first session. The other six participants performed with the weight of $0.450 \mathrm{~kg}$ followed by $0.250 \mathrm{~kg}$ in their first session.

\section{Data analysis}

The data were analyzed offline using MATLAB (Version R2016b, MathWorks, USA). Force/Torque data and laser displacement data of thumb were lowpass filtered at $15 \mathrm{~Hz}$ using second-order, zero phase lag Butterworth filter. In each trial, the data between $2 \mathrm{~s}$ and 5 s were taken for analysis to avoid start and end effects.

The normal and tangential force data collected from the individual fingertips and the thumb were averaged over the time samples, trials, and participants for each condition separately, and the standard errors of the mean were computed.

\section{Statistics}

All Statistical analyses were performed using R. Two-way repeated-measures ANOVA was performed on the average normal force with the two factors being loads (4 levels: $0.150 \mathrm{~kg}, 0.250 \mathrm{~kg}, 0.350 \mathrm{~kg}, 0.450 \mathrm{~kg}$ ) and fingers (4 levels: index, middle, ring, little). Since the thumb normal force is dependent on the normal forces of the index, middle, ring, and little fingers, a separate one-way repeated measures ANOVA was performed on the thumb normal force with the factor as loads (4 levels: $0.150 \mathrm{~kg}, 0.250 \mathrm{~kg}, 0.350 \mathrm{~kg}$, $0.450 \mathrm{~kg}$ ). Another two-way repeated-measures ANOVA was performed on the average tangential force with the factors being loads (4 levels: $0.150 \mathrm{~kg}, 0.250 \mathrm{~kg}, 0.350 \mathrm{~kg}, 0.450 \mathrm{~kg}$ ) and fingers (5 levels: index, middle, ring, little, thumb). Sphericity test was done on the data, and the number of degrees of freedom was adjusted by Huynh-Feldt ( $\mathrm{H}-\mathrm{F})$ criterion wherever required. Pairwise post hoc tukey tests were performed to examine the significance within factors. Further, we performed equivalence tests for all the non-different pairs. The statistical equivalence was tested using the two one-sided t-tests (TOST) approach $^{22}$ for a desired statistical power of $95 \%$. The smallest effect size of interest (SESOI) was chosen as the equivalence bounds. 


\section{Results}

\section{Task performance}

All the participants were able to trace the horizontal target line shown on the monitor within the error margin during all four loading conditions $(0.150 \mathrm{~kg}, 0.250 \mathrm{~kg}, 0.350 \mathrm{~kg}$, and $0.450 \mathrm{~kg}$ ) as shown in Supplementary Fig. S1. Root mean squared error (RMSE) on the thumb displacement data was computed for the four different loads and is shown in Table 1. Throughout the trial, the participants attempted to maintain the handle in static equilibrium during all four loading conditions by positioning the bubble at the center of the bull's eye. Therefore, the average net tilt angles for the different loading conditions were found to be less than one degree, as shown in Table 1. Thus, the participants could trace the target line with minimal vertical displacement and minimal tilt during all trials in all loading conditions.

Table 1 Root Mean Square Error on the thumb displacement data and Net tilt angle. The table shows the average net tilt angle measured in degrees and root mean square error (RMSE) in $\mathrm{cm}$ on the thumb displacement data with standard deviation for the four different loads $0.150 \mathrm{~kg}, 0.250 \mathrm{~kg}, 0.350 \mathrm{~kg}$, and $0.450 \mathrm{~kg}$

\begin{tabular}{|c|c|c|}
\hline $\begin{array}{c}\text { Additional Loads } \\
(\mathbf{k g})\end{array}$ & $\begin{array}{c}\text { Net tilt angle } \\
\text { (degrees) } \\
(\text { mean } \pm \text { SD) }\end{array}$ & $\begin{array}{c}\text { RMSE on the thumb displacement data } \\
\text { (cm) } \\
(\text { mean } \mathbf{S D})\end{array}$ \\
\hline 0.150 & $0.58 \pm 0.22$ & $0.0215 \pm 0.0054$ \\
\hline 0.250 & $0.73 \pm 0.19$ & $0.0246 \pm 0.0066$ \\
\hline 0.350 & $0.70 \pm 0.15$ & $0.0240 \pm 0.0071$ \\
\hline 0.450 & $0.81 \pm 0.23$ & $0.0325 \pm 0.0185$ \\
\hline
\end{tabular}

\section{Normal forces of the individual fingers and thumb during different loads}

The normal forces of the ring and little fingers were found to be statistically comparable with the addition of external loads of $0.150 \mathrm{~kg}, 0.250 \mathrm{~kg}$, and $0.350 \mathrm{~kg}$. However, when an external load of $0.450 \mathrm{~kg}$ was added, the little finger normal force was found to be statistically greater than the ring finger normal force and thus supporting MAH.

We observed a main effect of the factor loads $\left(F_{(2.73,30.03)}=8.571 ; p<0.001, \eta^{2}{ }_{p}=0.43\right)$ when a two-way repeated-measures ANOVA was performed on the absolute normal force with the factors as loads and fingers. It was found that the normal forces of the individual fingers (excluding the thumb) under the loading condition of $\mathbf{0 . 4 5 0} \mathbf{k g}$ were statistically $(\mathrm{p}<0.001)$ greater than the normal forces produced under

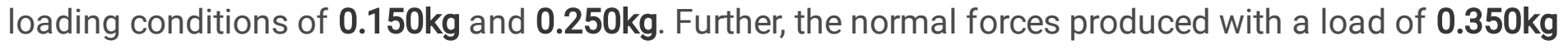
were statistically $(p<0.05)$ greater than the normal force produced with a load of $0.150 \mathbf{k g}$. In addition to this, there was a significant effect of the fingers $\left(F_{(3,33)}=181.921 ; p<0.001, \eta^{2}{ }_{p}=0.94\right)$ corresponding to a statistically $(p<0.001)$ higher normal force by the little finger than the index, middle and ring fingers on 
loading (refer Fig.2). Also, the normal force of the ring finger was statistically greater than the radial fingers.

Meanwhile, we also found that the ulnar finger normal forces were non-different when external loads of masses $0.150 \mathrm{~kg}\left(\mathrm{t}(11)=-1.129, \mathrm{p}=0.283, \mathrm{~d}_{\mathrm{z}}=0.32\right), 0.250 \mathrm{~kg}\left(\mathrm{t}(11)=-0.978, \mathrm{p}=0.349, \mathrm{~d}_{\mathrm{z}}=0.28\right)$ and $\mathbf{0 . 3 5 0} \mathbf{k g}\left(\mathrm{t}(11)=-1.454, \mathrm{p}=0.174, \mathrm{~d}_{\mathrm{z}}=0.41\right)$ were employed. Therefore, by using TOST procedure on the

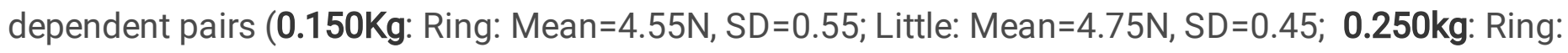
Mean=4.84N, SD=0.90; Little: Mean=5.07N, SD=0.50; 0.350kg : Ring: Mean=5.29N, SD=0.78; Little: Mean $=5.70 \mathrm{~N}, S D=0.58)$, it was confirmed that the ulnar fingers normal forces were statistically equivalent (0.150kg: $\mathrm{t}(11)=2.473, \mathrm{p}=0.0155 ; \mathbf{0 . 2 5 0} \mathbf{k g}: \mathrm{t}(11)=2.625, \mathrm{p}=0.0118 ; 0.350 \mathrm{~kg}: \mathrm{t}(11)=2.148$, $p=0.0274)$ with the observed effect size that falls within the equivalence bounds of $\Delta_{\mathrm{L}}=-1.04$ and $\Delta_{U}=1.04$ under the loads of $0.150 \mathrm{~kg}, 0.250 \mathrm{~kg}$ and $0.350 \mathrm{~kg}$.

The interaction loads $x$ fingers was significant $\left(F_{(3.96,43.56)}=18.538 ; p<0.001, \eta^{2}{ }_{p}=0.62\right)$ reflecting the fact that the ulnar finger normal forces of $\mathbf{0 . 4 5 0} \mathbf{k g}$ (Ring: $5.03 \mathrm{~N}$, Little: $6.94 \mathrm{~N}$ ), $0.350 \mathrm{~kg}$ (Ring: $5.29 \mathrm{~N}$, Little: $5.70 \mathrm{~N}), 0.250 \mathrm{~kg}$ (Ring: $4.84 \mathrm{~N}$; Little: $5.07 \mathrm{~N})$, were statistically $(\mathrm{p}<0.001)$ greater than the radial finger

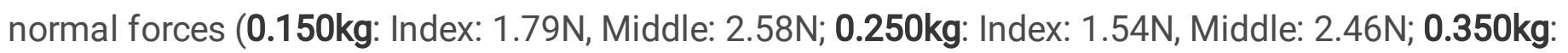

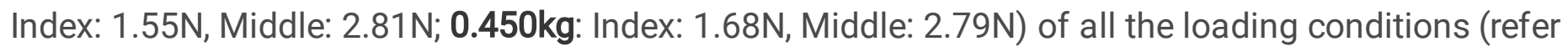
Fig.3).

While, the pairwise post hoc tukey tests confirmed that the little finger normal force $(6.94 \mathrm{~N})$ of $0.450 \mathrm{~kg}$ was statistically $(p<0.001)$ greater than the ulnar finger normal forces $(0.150 \mathrm{~kg}$ : Ring: $4.55 \mathrm{~N}$, Little: $4.75 \mathrm{~N}$; 0.250kg: Ring: $4.84 \mathrm{~N}$, Little: $5.07 \mathrm{~N} ; 0.350 \mathrm{~kg}$ : Ring: $5.29 \mathrm{~N}$, Little: $5.70 \mathrm{~N}(\mathrm{p}<0.01))$ of the remaining loads

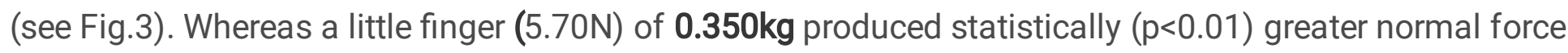
than the ring finger $(4.55 \mathrm{~N})$ under $0.150 \mathrm{~kg}$ of load.

One-way repeated-measures ANOVA was performed on the thumb normal force, which showed a significant effect of the factor loads $\left(F_{(2.69,88.90)}=9.411 ; p<0.001, \eta_{p}^{2}=0.46\right)$. Under the loading of $0.450 \mathrm{~kg}$, the thumb normal force $(16.50 \mathrm{~N})$ was found to be statistically greater than under loadings of $0.150 \mathrm{~kg}(13.73 \mathrm{~N}, \mathrm{p}<0.01)$ and $\mathbf{0 . 2 5 0 k g}(13.97 \mathrm{~N}, \mathrm{p}<0.05)$ (see Supplementary Fig.S2).

\section{Tangential forces of individual fingers during different loads}

In the case of the tangential forces, a two-way repeated-measures ANOVA with the factors as loads $\left(\mathrm{F}_{(3,}\right.$ $\left.33)=390.575 ; p<0.001, \eta_{p}^{2}=0.97\right)$ and fingers $\left(F_{(4,44)}=44.205 ; p<0.001, \eta_{p}^{2}=0.80\right)$ showed significant effect of the factor loads corresponding to a statistically greater tangential force with the use of $0.450 \mathrm{~kg}$ 
than with the use of $0.150 \mathrm{~kg}(\mathrm{p}<0.001), 0.250 \mathrm{~kg}(\mathrm{p}<0.001)$ and $0.350 \mathrm{~kg}(\mathrm{p}<0.05)$. In addition, a significant effect of the factor fingers confirmed that the little finger tangential force was statistically $(p<0.001)$ greater than the index, middle, and ring finger tangential forces on loading.

In addition to this, on performing the pairwise post hoc tukey test, it was confirmed that the little finger tangential force $(0.150 \mathrm{~kg}: 2.03 \mathrm{~N}, \mathbf{0 . 3 5 0} \mathbf{k g}: 2.80 \mathrm{~N})$ was non-different from the ring finger tangential force during the employment of $0.150 \mathrm{~kg}(1.64 \mathrm{~N})$ and $0.350 \mathrm{~kg}(2.26 \mathrm{~N})$. TOST procedure performed on these dependent pairs confirmed that these comparisons were not statistically equivalent as well. However, little finger tangential force $(\mathbf{0 . 4 5 0} \mathbf{k g}: 3.22 \mathrm{~N} ; \mathbf{0 . 2 5 0} \mathbf{k g}: 2.54 \mathrm{~N})$ was statistically greater than the ring finger

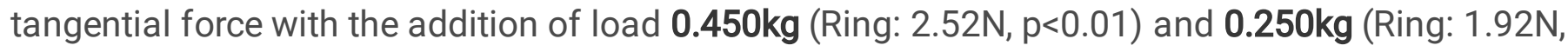
p<0.05) (refer Fig.4).

Further, interaction effect of loads $x$ fingers was significant $\left(F_{(12,132)}=5.857 ; p<0.001, \eta^{2}{ }_{p}=0.34\right)$ reflecting the fact that the little finger tangential forces $(3.22 \mathrm{~N})$ due to the use of $0.450 \mathrm{~kg}$ was statistically greater than the ulnar fingers tangential forces $(\mathbf{0 . 1 5 0 k g}$ : Ring: $1.64 \mathrm{~N}, p<0.01$, Little: $2.03 \mathrm{~N}, p<0.001 ; \mathbf{0 . 2 5 0} \mathbf{k g}$ : Ring:1.92N, $p<0.001$, Little: $2.54 \mathrm{~N}, p<0.05 ; 0.350 \mathrm{~kg}$ : Ring: $2.26 \mathrm{~N}, \mathrm{p}<0.001)$ caused due to the other loads (refer Supplementary Fig.S3).

\section{Discussion}

The main objective of the present study was to investigate whether the applicability of MAH is specific to the perceived nature of the task and is not dependent solely on task parameters like the total weight of the handle or the compensatory torque requirement. We tested the manifestation of MAH in our paradigm by systematically increasing the weight of the handle by adding external loads at the bottom of the handle below its center of mass. The weight of our current handle with the minimal loading condition exceeded the weight of the handle in our previous study ${ }^{19}$. So, we hypothesized that MAH would be supported in all our loading conditions. Contrary to our expectation, we found that the ring and little finger normal forces were statistically comparable with the addition of $0.150 \mathrm{~kg}, 0.250 \mathrm{~kg}$, and $0.350 \mathrm{~kg}$ loads. However, we noticed that MAH was supported for the external load of $0.450 \mathrm{~kg}$. We discussed the implications of these findings in the following paragraphs.

Ulnar finger normal forces were examined under four different external loading conditions i.e., $0.150 \mathrm{~kg}$, $0.250 \mathrm{~kg}, 0.350 \mathrm{~kg}$ and $0.450 \mathrm{~kg}$. In our previous study ${ }^{19}$, with a similar unsteady thumb platform as used in the current study, the ulnar fingers produced comparable normal forces for a handle mass of $0.535 \mathrm{~kg}$. With the minimal external load of $0.150 \mathrm{~kg}$, the total mass of the handle would become $0.600 \mathrm{~kg}$ (above $0.535 \mathrm{~kg}$ studied in our previous study). So, our expectation was that MAH would be supported for all the loading conditions. In contrast to our expectation, the little finger produced statistically comparable normal forces to the ring finger for $0.150 \mathrm{~kg}$ load. With further increase in the external loadings with masses $0.250 \mathrm{~kg}$ and $0.350 \mathrm{~kg}$, the ulnar fingers exhibited statistically comparable normal forces. However, this trend did not hold true when the external load was increased to $0.450 \mathrm{~kg}$, wherein the little finger exerted a statistically greater normal force than the ring finger. 
Unlike the other studies on grasping with eccentrically loaded manipulanda, the current study involved maintaining a constant minimal tangential force by the thumb (approximately $1 \mathrm{~N}$ ) at different loading conditions (see Supplementary Fig.S4). Therefore, with an increase in the total mass of the handle by adding an external load of $0.450 \mathrm{~kg}$ (comparatively larger than the mass of other loads employed in the present study), the virtual finger had to share greater tangential force to maintain the vertical equilibrium causing a greater pronation torque (counter-clockwise direction from the participants viewpoint). This, in turn, necessitated a progressively greater compensatory supination moment to maintain the rotational equilibrium. Since the design of the handle prevents the thumb from contributing further to the supination moment, the ulnar fingers are required to compensate with their normal forces. The little finger tends to produce greater normal force among the ulnar fingers than the ring finger, thus supporting MAH.

A natural question is whether the applicability of mechanical advantage depends on employing heavy masses while grasping. If that had been true, then MAH would have been supported when a large external load of $2 \mathrm{~kg}$ was suspended at a distance of $1.9 \mathrm{~cm}$ from the center of mass (COM) of the handle (for a torque magnitude of $-0.375 \mathrm{Nm}$ ) in the grasping study investigating the contribution of peripheral and central fingers ${ }^{16}$. However, they found that ulnar fingers normal forces were non-different for this large load. Eventually, with a systematic increase in the compensatory supination torque magnitude $(0.750 \mathrm{Nm}$, $1.125 \mathrm{Nm}$, and $1.50 \mathrm{Nm}$ ), the little finger gradually started producing more normal force than the ring finger and validated the MAH.

In another study investigating the role of grasp force magnitude during multi-finger prehension ${ }^{21}$, when an external load of mass $0.160 \mathrm{~kg}$ (much lesser than $2 \mathrm{~kg}$ ) was suspended from a handle of mass $0.415 \mathrm{~kg}$ eccentrically at a distance of $9.5 \mathrm{~cm}$ from COM, MAH was supported. This result triggers another question as to whether the support for MAH depends on suspending the external load at large moment arms from COM of the handle? From the results of the multi-finger prehension study ${ }^{21}$, it was apparent that the applicability of mechanical advantage depends on using higher moment arms for the external load. Our current result forces us to re-evaluate this conclusion, as MAH was supported even when an external load of $0.450 \mathrm{~kg}$ was suspended directly below the COM of the handle (having zero moment arm). This suggests that apart from the mass of the external load and moment arm of the suspended load, a latent factor governs the applicability of mechanical advantage. In other words, our data suggest that the applicability of the principle of mechanical advantage in biological systems depends not only on the mass or moment arm of the suspended load or both but also on an abstract sense of challenge with which the participant perceives the task. Whether or not this sense of challenge stems from not being familiar with lifting asymmetric objects with a minimal tangential force of the thumb in day-to-day life warrants further research.

In the prehension study evaluating the effect of grasp force magnitude ${ }^{21}$, the challenging aspect of the task might have been using an unusually high moment arm, thus allowing MAH to manifest. In a recent study ${ }^{23}$ using a handle similar to the current study, the task was to trace trapezoid and inverted trapezoid patterns by displacing the thumb platform $1.5 \mathrm{~cm}$ above and below the HOME position. The mechanical 
advantage hypothesis was supported during the inverted trapezoid condition when the movable thumb platform was held steady while tracing the static portion $1.5 \mathrm{~cm}$ below the HOME (at the level below the center of the ring finger sensor). The carpometacarpal joint (CMC) of the thumb has a restricted range of motion in the downward direction ${ }^{24}$ (flexion or radial adduction). Therefore the task of maintaining the handle in static equilibrium with a movable thumb platform at the level below the center of the ring finger sensor might have been quite challenging to perform. We suggest that this sense of challenge would have caused the little finger to share greater normal force than the ring finger. Following a similar rationale, in the current study, perhaps the task became fairly demanding, as the requirement was to produce compensatory supination torque with only the normal forces of the ulnar fingers. This was a direct effect of restricting the thumb tangential force to a constant minimal magnitude and essentially rendering it much less consequential in the supination torque production. Simultaneously, this also amplified the role of the ulnar fingers in the compensatory torque production. This sense of challenge inherent in the nature of the task progressively increased with increase in the external loads.

To further elucidate our result, it is important to emphasize that in the previous studies on object manipulation that introduced external torques to the handle, there was no restriction in the distribution of tangential forces among the fingers and the thumb while grasping. The tangential force of the thumb would have greatly contributed to the supination torque in addition to the normal forces of the ulnar fingers. This was evident from the previous study ${ }^{16}$, where the thumb tangential force increased during the supination efforts. Hence, the participants might have been able to share comparable normal forces by the ring and little fingers even with a larger load $(2 \mathrm{~kg})$ and with a greater torque magnitude of $0.375 \mathrm{Nm}$ than in the current study.

In contrast, in our current study, the tangential force of the thumb was restricted to approximately $1 \mathrm{~N}$ by placing the thumb on a freely movable platform of mass $0.100 \mathrm{~kg}$ for all the loading conditions. This essentially creates a situation wherein the ulnar fingers are forced to contribute greatly to the compensatory supination torque. We posit that such a constraint in the tangential force contribution of the thumb presents a great sense of challenge to the participant. This is most exemplified under $0.450 \mathrm{~kg}$ external load. Note that this load is much less than the $2 \mathrm{~kg}$ load where MAH was not supported. We strongly believe that this sense of challenge might have triggered the use of mechanical advantage by the central nervous system (CNS). In our study, the phrase 'sense of challenge' refers to a kind of task difficulty that is not merely dictated by the external loads and torques. The difficulty faced by the performer during the task could be due to the biomechanical constraint as in the previous study ${ }^{23}$, or it could be due to the perceived nature of the task.

In the present study, the participants could complete the task under loads of $0.150 \mathrm{~kg}, 0.250 \mathrm{~kg}$, and $0.350 \mathrm{~kg}$ (resulting in the supination torques of $0.22 \mathrm{Nm}, 0.23 \mathrm{Nm}$, and $0.25 \mathrm{Nm}$, respectively), which might not be challenging enough than a load of mass $0.450 \mathrm{~kg}$ (refer Fig. 5 and Supplementary Fig.S5). As under a load of mass $0.450 \mathrm{~kg}$, the sense of challenge from the nature of the task becomes the most exemplified. The controller perhaps would have attempted to choose an energy-efficient option of adopting the mechanical advantage principle of employing the little finger with a longer moment arm for 
normal force to produce greater force. From a neural perspective, we believe that the mechanisms through which mechanical advantage is enabled might have involved greater activation of the little finger motor units.

In a study on producing maximum voluntary contraction $\mathrm{MVC}^{25}$, when the target finger is the little finger, the force produced by the little finger was found to be well above the adjacent ring finger force. Analogously, since our study involved very strong voluntary grasping of the handle for the external loading condition of $0.450 \mathrm{~kg}$, greater activation of the little finger motor units could have caused a greater force in the little finger than the ring finger, thus enabling optimal distribution of forces within the ulnar fingers in line with the MAH. This is also supported by the study ${ }^{26}$ wherein they found that that the magnitude of force produced due to the little finger motor units under the ring finger was almost twothirds of the force produced under little finger during voluntary grasping. Since we have not measured the actual activation pattern of the individual motor units, further research is required to tease out the underlying neural mechanisms through which mechanical advantage is manifested. Taken together, our results suggest that the applicability of the mechanical advantage hypothesis depends not only on the torque requirement or the total mass of the object but also on the kind of task performed and the sense of challenge associated with it.

\section{Concluding Comments}

The current study was performed to validate whether the mechanical advantage hypothesis is taskspecific and investigate the kind of task the MAH depends on. A five-finger prehensile handle with an unsteady thumb platform was utilized for analyzing the applicability of MAH. The mass of the handle was systematically increased by using additional external loads of mass $0.150 \mathrm{~kg}, 0.250 \mathrm{~kg}, 0.350 \mathrm{~kg}$, and $0.450 \mathrm{~kg}$. Ulnar fingers exerted a comparable normal force with the external loads of mass $0.150 \mathrm{~kg}$, $0.250 \mathrm{~kg}$, and $0.350 \mathrm{~kg}$. However, the mechanical advantage hypothesis was supported with a load of $0.450 \mathrm{~kg}$. With the addition of greater mass, under the constraint of minimal thumb tangential force, establishing static equilibrium by the ulnar fingers becomes challenging. Therefore, we conclude that the $\mathrm{MAH}$ is task-specific in nature and depends on how challenging the task is.

\section{Declarations}

\section{Acknowledgments}

We thank the Department of Science \& Technology, Government of India, for supporting this work, vide Reference Nos SR/CSRI/97/2014 \& DST/CSRI/2017/87 under Cognitive Science Research Initiative (CSRI) (awarded to Varadhan SKM), American Express (funding awarded to DART lab, IIT Madras) and "Women leading IIT Madras (WLI)" grant (awarded to Banuvathy Rajakumar). The funders had no role in study design, data collection and analysis, decision to publish, or preparation of the manuscript.

\section{Author Contributions Statement}


Conceptualization - BR, VSKM; Methodology - BR, SD, VSKM; Formal Analysis - BR; Writing Original Draft - BR; Writing, Review, and Editing -BR, SD, VSKM.

\section{Competing interests}

The authors declare no competing interests.

\section{Data Availability}

We plan to publish a data descriptor article along with this manuscript. Hence the data will be made available in due course of time.

\section{References}

1. Schneider, T., Buckingham, G. \& Hermsdörfer, J. Torque planning errors affect the perception of object properties and sensorimotor memories during object manipulation in uncertain grasp situations. Journal of Neurophysiology 121, (2019).

2. Santello, M. \& Soechting, J. F. Force synergies for multifingered grasping. Exp Brain Res 133, 457467 (2000).

3. Zatsiorsky, V. M., Gao, F. \& Latash, M. L. Prehension synergies: Effects of object geometry and prescribed torques. Exp Brain Res 148, 77-87 (2003).

4. Zatsiorsky, V. M., Gao, F. \& Latash, M. L. Finger force vectors in multi-finger prehension. J Biomech 36, 1745-1749 (2003).

5. Johansson, R. S. \& Westling, G. Roles of glabrous skin receptors and sensorimotor memory in automatic control of precision grip when lifting rougher or more slippery objects. Exp Brain Res 56, 550564 (1984).

6. Winstein, C. J., Abbs, J. H. \& Petashnick, D. Influences of object weight and instruction on grip force adjustments. Exp Brain Res 87, 465-469 (1991).

7. Cadoret, G. \& Smith, A. M. Friction, not texture, dictates grip forces used during object manipulation. J Neurophysio/ 75, 1963-1969 (1996).

8. Cole, K. J. \& Johansson, R. S. Friction at the digit-object interface scales the sensorimotor transformation for grip responses to pulling loads. Exp Brain Res 95, 523-532 (1993).

9. Aoki, T., Latash, M. L. \& Zatsiorsky, V. M. Adjustments to Local Friction in Multifinger Prehension. J Mot Behav 39, 276-290 (2007).

10. Zatsiorsky, V. M., Gao, F. \& Latash, M. L. Prehension Stability: Experiments With Expanding and Contracting Handle. J Neurophysio/ 95, 2513-2529 (2006). 
11. Slota, G., Latash, M. \& Zatsiorsky, V. Tangential Finger Forces Use Mechanical Advantage During Static Grasping. Journal of applied biomechanics 28, 78-84 (2012).

12. Solnik, S., Zatsiorsky, V. M. \& Latash, M. L. Internal Forces during Static Prehension: Effects of Age and Grasp Configuration. J Mot Behav 46, 211-222 (2014).

13. Buchanan, T. S., Rovai, G. P. \& Rymer, W. Z. Strategies for muscle activation during isometric torque generation at the human elbow. Journal of Neurophysiology 62, 1201-1212 (1989).

14. Prilutsky, B. I. Coordination of two- and one-joint muscles: functional consequences and implications for motor control. Motor Control 4, 1-44 (2000).

15. Zhang, W., Olafsdottir, H. B., Zatsiorsky, V. M. \& Latash, M. L. Mechanical Analysis and Hierarchies of Multi-digit Synergies during Accurate Object Rotation. Motor Control 13, 251-279 (2009).

16. Zatsiorsky, V. M., Gregory, R. W. \& Latash, M. L. Force and torque production in static multifinger prehension: biomechanics and control. I. Biomechanics. Biological Cybernetics 87, 50-57 (2002).

17. Olafsdottir, H., Zhang, W., Zatsiorsky, V. M. \& Latash, M. L. Age-related changes in multifinger synergies in accurate moment of force production tasks. J App/ Physio/ 102, 1490 (2007).

18. Shim, J. K., Latash, M. L. \& Zatsiorsky, V. M. Finger coordination during moment production on a mechanically fixed object. Exp Brain Res 157, 457-467 (2004).

19. Rajakumar, B. \& Skm, V. Comparable behaviour of ring and little fingers due to an artificial reduction in thumb contribution to hold objects. PeerJ (2020) doi:10.7717/peerj.9962.

20. MacKenzie, C. L. \& Iberall, T. The Grasping Hand. (Elsevier, 1994).

21. Niu, X., Latash, M. \& Zatsiorsky, V. Effects of Grasping Force Magnitude on the Coordination of Digit Forces in Multi-finger Prehension. Experimental brain research. Experimentelle Hirnforschung. Expérimentation cérébrale 194, 115-29 (2009).

22. Lakens, D. Equivalence Tests: A Practical Primer for $t$ Tests, Correlations, and Meta-Analyses. Social Psychological and Personality Science 8, 355-362 (2017).

23. Banuvathy, R. \& Varadhan, S. K. M. Distinct behavior of the little finger during the vertical translation of an unsteady thumb platform while grasping. Sci Rep 11, 21064 (2021).

24. Barakat, M., Field, J. \& Taylor, J. The range of movement of the thumb. HAND 8, (2013).

25. Zatsiorsky, V. M., Li, Z. M. \& Latash, M. L. Enslaving effects in multi-finger force production. Exp Brain Res 131, 187-195 (2000). 
26. Kilbreath, S. L., Gorman, R. B., Raymond, J. \& Gandevia, S. C. Distribution of the forces produced by motor unit activity in the human flexor digitorum profundus. The Journal of Physiology 543, 289-296 (2002).

\section{Figures}

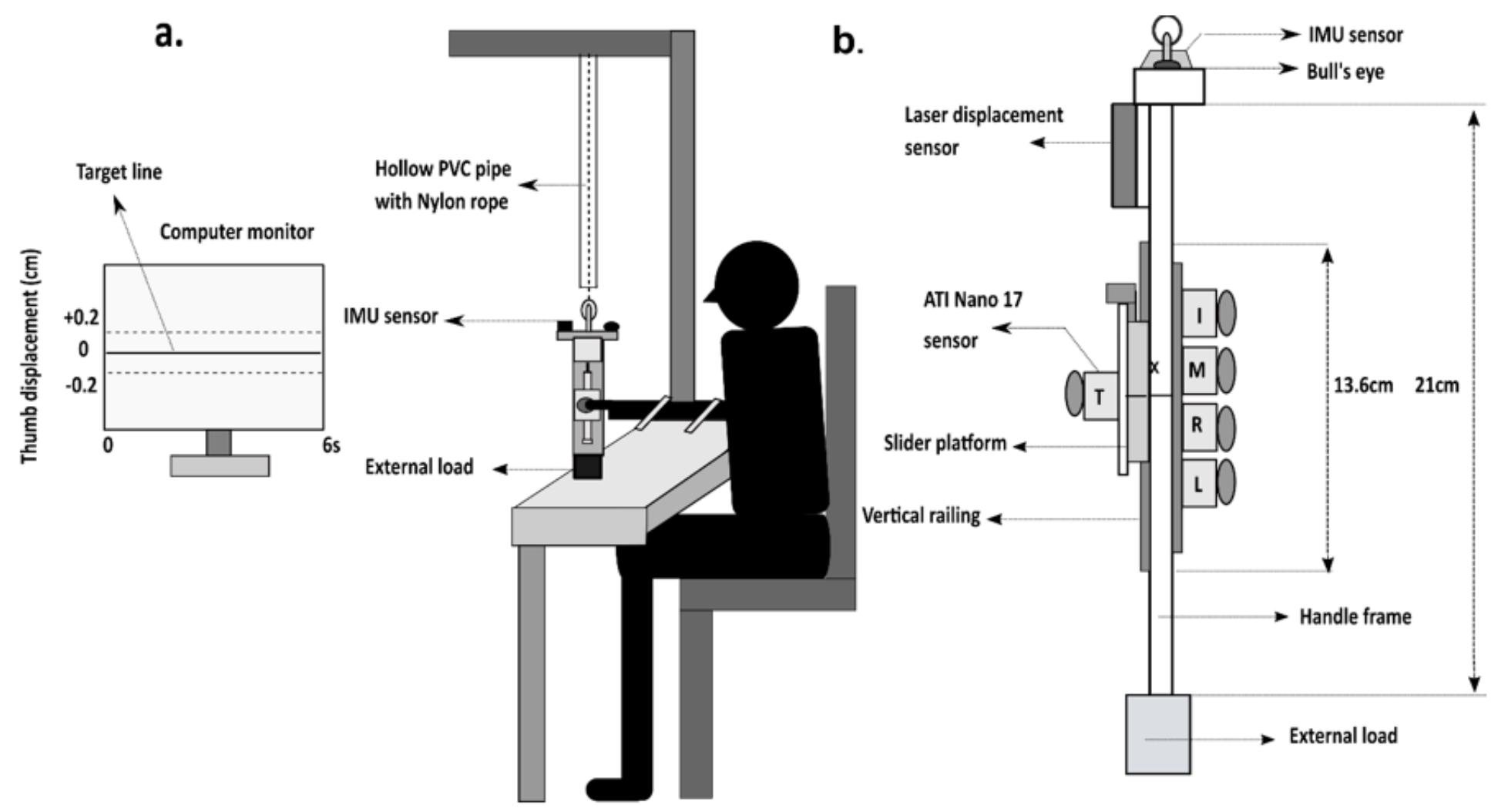

\section{Figure 1}

Schematic diagram of the experimental setup and five-finger prehensile handle. A. Experimental setup with the participant holding the handle at a distance of $1.5 \mathrm{~m}$ away from the computer monitor. The handle was suspended from a wooden support using a nylon rope housed within the hollow PVC pipe to restrict the lateral movements of the handle. The solid horizontal target line was shown on the computer monitor with two dashed lines that represented an acceptable error margin. B. Schematic diagram of the experimental handle. The aluminum handle frame $(21 \times 1 \times 3) \mathrm{cm}$ with five fingertip force (ATI Nano 17) sensors, laser displacement sensor, and orientation measuring sensor (IMU) are shown. The grip aperture of the handle is $6.2 \mathrm{~cm}$. External loads of $0.150 \mathrm{~kg}, 0.250 \mathrm{~kg}, 0.350 \mathrm{~kg}$, and $0.450 \mathrm{~kg}$ were attached at the bottom of the handle (i.e.) below the center of mass (represented as ' $X$ ') of the handle. I, $M, R, L, T$ represents Index, Middle, Ring, Little, and Thumb. 


\section{Average Normal force of all fingers except thumb at different load condtions}

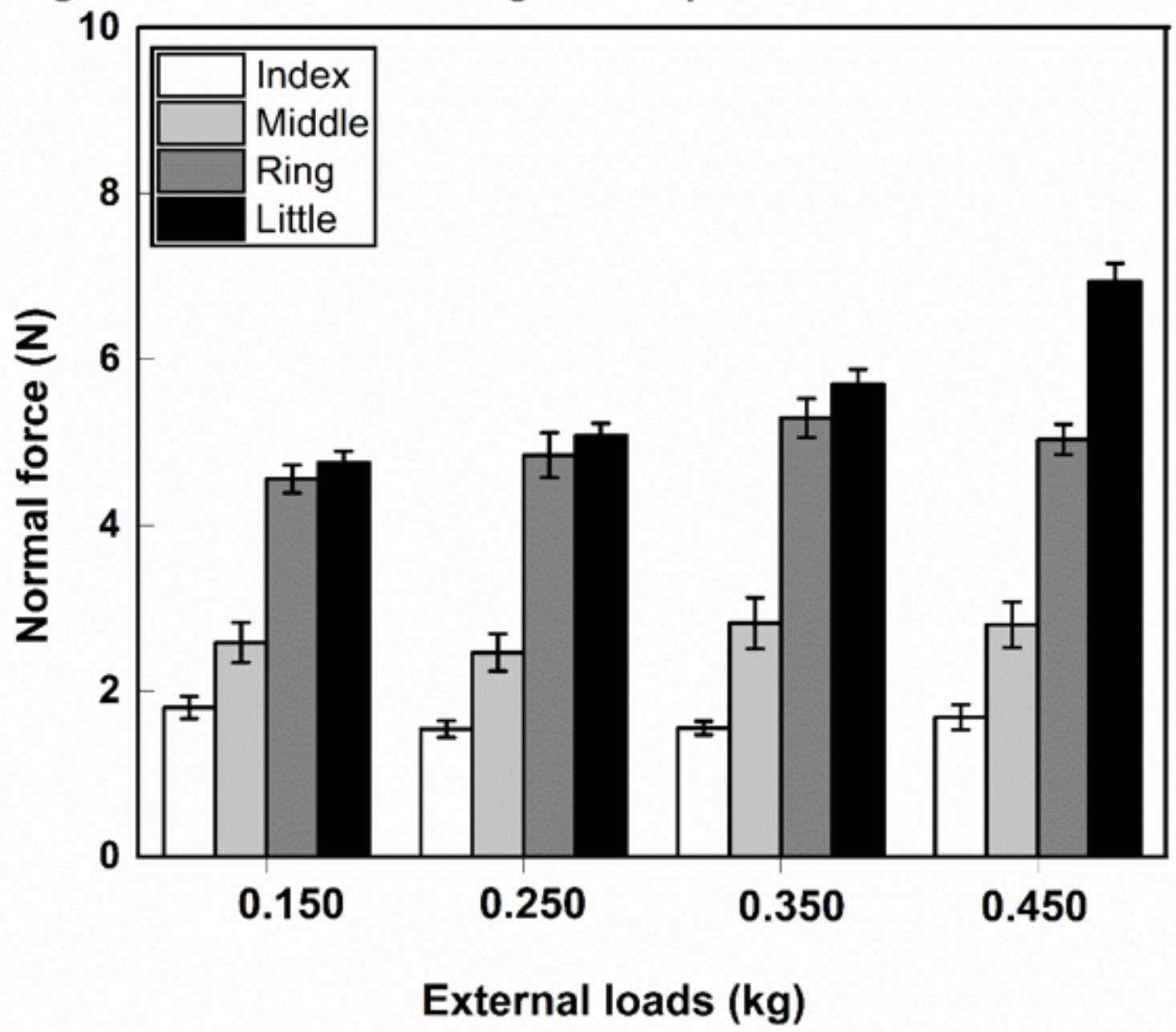

Figure 2

Average Normal force of Index, Middle, Ring, and Little fingers under different loading conditions. Little finger normal force (represented in black) was found to be statistically greater than the ring finger normal force (represented in dark shaded grey) in the $0.450 \mathrm{~kg}$ loading condition. The ulnar finger normal forces were found to be statistically equivalent under remaining loading conditions. The columns and bars indicate means and standard errors of means. 


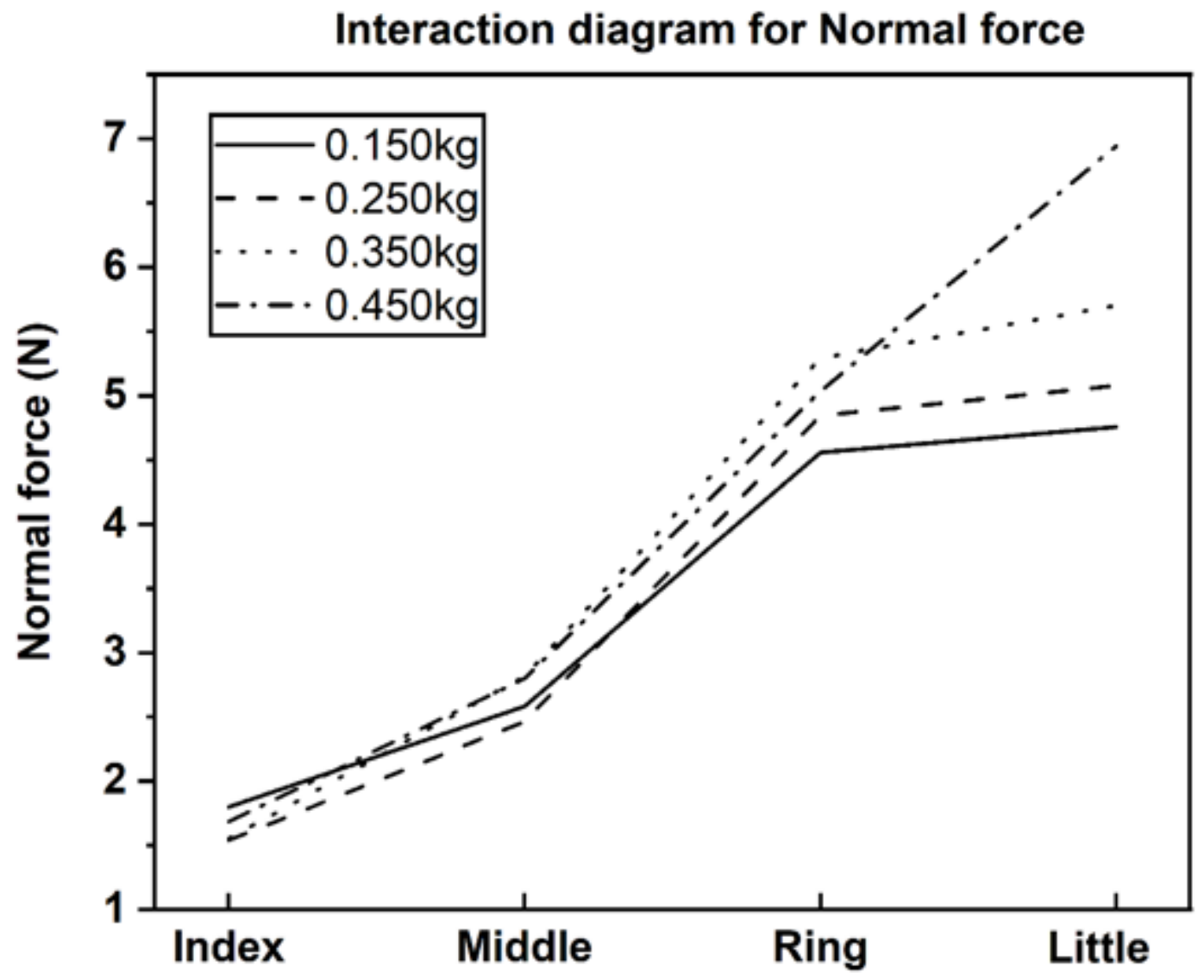

Fingers

Figure 3

Interaction between loads and finger normal forces The pairwise post hoc tukey tests confirmed that the ulnar finger normal forces of $0.450 \mathrm{~kg}$ (Ring: $5.03 \mathrm{~N}$, Little: $6.94 \mathrm{~N}$ ) $0.350 \mathrm{~kg}$ (Ring: $5.29 \mathrm{~N}$, Little: $5.70 \mathrm{~N}$ ), $0.250 \mathrm{~kg}$ (Ring: $4.84 \mathrm{~N}$; Little: $5.07 \mathrm{~N}$ ) were statistically greater than the radial finger normal forces (0.150kg: Index: $1.79 \mathrm{~N}$, Middle: $2.58 \mathrm{~N} ; 0.250 \mathrm{~kg}$ : Index: $1.54 \mathrm{~N}$, Middle: $2.46 \mathrm{~N} ; 0.350 \mathrm{~kg}$ : Index: $1.55 \mathrm{~N}$, Middle: $2.81 \mathrm{~N} ; 0.450 \mathrm{~kg}$ : Index: $1.68 \mathrm{~N}$, Middle: $2.79 \mathrm{~N}$ ) of all the loading conditions. 


\section{Average Tangential Force at different load conditions}

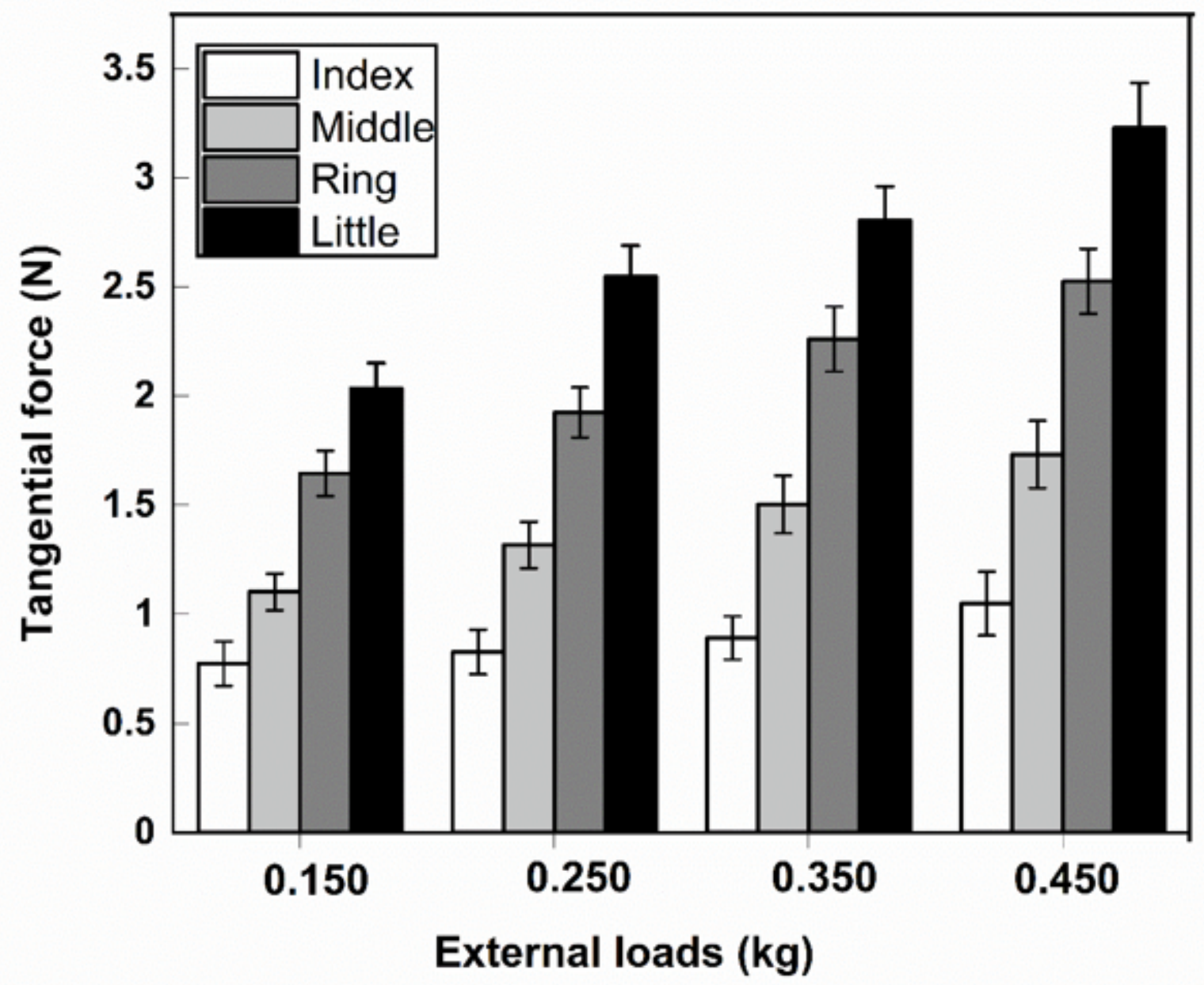

Figure 4

Average tangential force of Index, Middle, Ring, and Little under different loading conditions. Little finger tangential force $(0.250 \mathrm{~kg}: 2.54 \mathrm{~N} ; 0.450 \mathrm{~kg}: 3.22 \mathrm{~N})$ was found to be statistically greater than the ring finger tangential force $(0.250 \mathrm{~kg}: 1.92 \mathrm{~N} ; 0.450 \mathrm{~kg}: 2.52 \mathrm{~N})$ under $0.250 \mathrm{~kg}$ and $0.450 \mathrm{~kg}$ loading conditions. In particular, the little finger tangential forces $(3.22 \mathrm{~N})$ of $0.450 \mathrm{~kg}$ was statistically greater than the little finger tangential forces (0.150kg: Little: $2.03 \mathrm{~N} ; 0.250 \mathrm{~kg}$ : Little: $2.54 \mathrm{~N})$ of $0.150 \mathrm{~kg}$ and $0.250 \mathrm{~kg}$. The columns and bars indicate the means and standard errors of means. 


\section{Average Normal force of the Ulnar fingers at different supination torques}

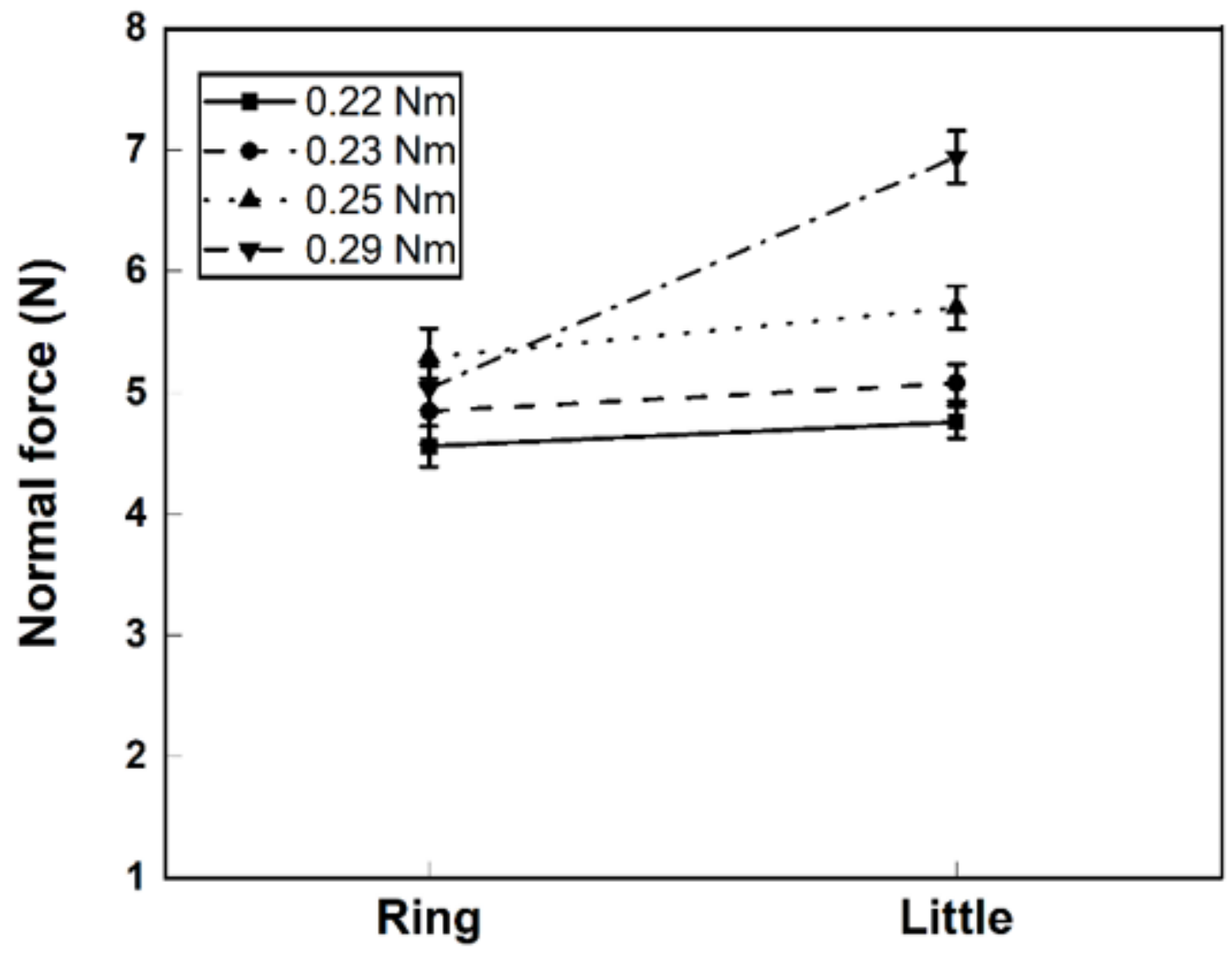

Fingers

Figure 5

Normal forces of ring and little fingers for supination efforts under different loading

conditions/supination torque requirement. For $0.29 \mathrm{Nm}$ torque requirement (represented by dashes with dots), the normal force of little finger was found to be statistically greater than ring finger normal force. In all the other conditions, ring and little fingers produced comparable normal force. The bars indicate standard errors of means.

\section{Supplementary Files}

This is a list of supplementary files associated with this preprint. Click to download.

- SupplementarymaterialRajakumarDuttaSKM.docx 